\title{
COMPORTAMIENTO FRENTE A LA ACCION DEL FUEGO DEL SISTEMA CONSTRUCTIVO DRAGADOS-PLASTBAU
}

\author{
Luis Miguel Elvira Martín, Laboratorio del Fuego. Dep. de Maderas, INIA \\ Fco. Javier Jiménez Peris, Laboratorio del Fuego, Dep. de Maderas, INIA \\ Jesús Rodriguez Santiago, Dragados y Construcciones, S. A.
}

\section{CONSIDERACIONES PREVIAS}

La resistencia al fuego de un elemento constructivo portante viene determinada por el tiempo, expresado en minutos, durante el cual es capaz de mantener las condiciones de estabilidad mecánica, aislamiento térmico, estanquidad a las llamas y ausencia de gases inflamables ante la acción de un incendio, según lo establecido en la norma NBE-CPI-82 (1); o dicho de otra forma, es el tiempo en que dicho elemento permanece cumpliendo sus funciones aún a pesar de la acción del fuego.

Su determinación puede llevarse a cabo por métodos teóricos o experimentales existiendo también tabulaciones en códigos y publicaciones especializadas que contienen el valor de la resistencia al fuego de los elementos constructivos comúnmente utilizados.

En el sistema Dragados-Plastbau, la combinación de materiales que forman sus componentes principales, muros y forjados, no ha permitido conocer el valor de su resistencia al fuego ni por procedimientos teóricos de cálculo ni mediante las tabulaciones antes indicadas, por lo que se ha procedido por la via experimental, mediante ensayos de laboratorio.

En la Tecnologia de Ensayos del Fuego y dentro del ámbito legislativo español, la Norma Básica de la Edificación-Condiciones de Protección contra Incendios en los Edificios NBE-CPI-82 (1) indica el "grado de resistencia al fuego" que debe presentar cada parte de un local o de la construcción, en función de los efectos que se presume podrian sufrir en caso de incendio.
Estos grados corresponden a elevaciones de temperatura en función del tiempo que se determinaron después de numerosas observaciones efectuadas en incendios reales.

La curva de estas elevaciones de temperatura en función del tiempo llamada "curva normalizada temperatura-tiempo" es prácticamente la misma en todos los paises que tienen normativa sobre Tecnología de Ensayos del Fuego en la construcción (2).

Ella está dada por la ecuación:

$T=T_{0}+345 \log _{10}(8 t+1)$

donde:

$t=$ tiempo, expresado en minutos.

$\mathrm{T}=$ temperatura a $10 \mathrm{~cm}$ de la muestra en el tiempo $t$, medida en $\circ \mathrm{C}$.

$\mathrm{T}_{\mathrm{o}}=$ temperatura inicial en $\circ \mathrm{C}$, constante para cada ensayo.

En el artículo 3.1.3. de la norma citada (1) se establecen los grados de clasificación de resistencia al fuego. En la normativa francesa se admite la clasificación 1/4 de hora mientras que en la española se eleva a $1 / 2$ hora el mínimo tiempo de cumplimiento para poder clasificar el elemento ensayado.

La respuesta de las muestras, que se ensayan a escala real, frente a la acción del fuego, se de- 
termina en función de los criterios siguientes (2):

a) Estabilidad mecánica.

b) Estanquidad a las llamas.

c) Aislamiento térmico.

d) Emisión de gases inflamables.

Así, en función de estos criterios, los elementos se juzgan de acuerdo a su grado de:

- Estabilidad al fuego - criterio a.

- Parallamas - criterio $a+b+d$.

- Cortafuegos - criterio $a+b+c+d$.

Sobre esta base y teniendo en cuenta la experiencia previa del Laboratorio del Fuego del INIA y de otros laboratorios extranjeros en este campo, se planteó un programa de ensayos para valorar el comportamiento frente al fuego de los muros y forjados del sistema DragadosPlastbau.

\section{MUESTRAS ENSAYADAS}

Se prepararon cinco muestras que reproducian a escala real los elementos principales del sistema y cuyas caracteristicas se resumen en las dos primeras columnas de la tabla 1 .

En primer lugar se ensayó un muro D-P \| con un número de pilares de hormigón armado repre- sentativo del utilizado habitualmente en los edificios. Posteriormente se ensayaron dos muros D-P I, uno de ellos con una capa de terminación de yeso de $2,0 \mathrm{~cm}$ de espesor, con el fin de conocer su comportamiento con y sin dicha protección adicional. Todos los muros se construyeron a partir de varios paneles unidos entre si mediante el solapo de sus mallas, lo que permitió reproducir las uniones de estos elementos tal y como se realizan en las construcciones reales.

Se ensayaron también dos forjados, el primero de los cuales correspondia a un forjado de cubierta D-P I con unas armaduras inferiores de refuerzo $\varnothing 6 / 0,20$ y una capa de terminación de yeso de $1,5 \mathrm{~cm}$ de espesor, fabricado a partir de varios paneles unidos mediante el solapo de sus mallas. El segundo forjado ensayado fue uno de hormigón armado tipo Cádiz con un canto y unas armaduras acordes con la separación entre los apoyos del horno en donde se ensayó.

\section{PROCEDIMIENTOS DE ENSAYO}

Los muros se ensayaron en un horno vertical de ladrillo refractario revestido en su interior con material aislante y con una boca útil de $2,90 \times$ $2,81 \mathrm{~m}$, dotado con tres quemadores de impulsión con una potencia de hasta $400.000 \mathrm{kcal} / \mathrm{h}$ cada uno, alimentados por propano. Los forjados se ensayaron en un horno horizontal de ladrillo refractario revestido en su interior con material aislante y con una boca útil de $3,70 \times 1,63 \mathrm{~m}$, dotado con dos quemadores de impulsión de ca-

TABLA 1

Resultados de los ensayos del fuego

\begin{tabular}{|c|c|c|c|c|c|}
\hline \multirow{2}{*}{ ENSAYO } & \multirow{2}{*}{ DIMENSIONES } & \multirow{2}{*}{ CARGAS } & \multirow{2}{*}{ RESUMEN DEL ENSAYO } & \multicolumn{2}{|c|}{ CLASIFICACION DEL ELEMENTO CONSTRUCTIVO } \\
\hline & & & & RESISTENCIA AL FUEGO & PARALLAMAS \\
\hline $\begin{array}{l}\text { MURO D-P.II } \\
\text { Pilares H.A. cada } 0,60 \mathrm{~m} \text {. } \\
\text { ( } 4 \varnothing 10 \text { y } E \emptyset 6 / 0,15) \\
\text { Vigas zuncho de Hormigon } \\
\text { en bordes sup. e inf. } \\
\text { ( } 4 \varnothing 8 \text { y } E \emptyset 6 / O, 15 \text { ) }\end{array}$ & $\begin{array}{l}H=3,40 \mathrm{~m} \\
b=3,30 \mathrm{~m} \\
h=0,28 \mathrm{~m}\end{array}$ & $13,6 \mathrm{t} / \mathrm{m}$ & $\begin{array}{l}\text { A los } 2 \mathrm{~h} .19 \mathrm{~min} \text {. se alcanzó un } \\
\Delta T=180^{\circ}(*) \text { prolongándose el ensayo } \\
\text { hasta los } 3 \mathrm{~h} \text {. }\end{array}$ & RF 120 & 180 \\
\hline $\begin{array}{l}\text { MURO D.-P.I } \\
\text { Capa de } 2 \mathrm{~cm} \text { de yeso }\end{array}$ & $\begin{array}{l}H=3,40 \mathrm{~m} \\
b=3,30 \mathrm{~m} \\
h=0,15 \mathrm{~m}\end{array}$ & $7,6 \mathrm{t} / \mathrm{m}$ & $\begin{array}{l}\text { A los } 2 \mathrm{~h} .19 \mathrm{~min} \text {. se alcanzó un valor medio } \\
\Delta T=140^{\circ}(*) \text { prolongándose el ensoyo } \\
\text { hasta las } 3 \mathrm{~h} \text {. }\end{array}$ & $\mathrm{RF} 120$ & 180 \\
\hline MURO D. -P.I & $\begin{array}{l}H=3,40 \mathrm{~m} \\
b=3,30 \mathrm{~m} \\
h=0,13 \mathrm{~m}\end{array}$ & $7,6 \mathrm{t} / \mathrm{m}$ & $\begin{array}{l}\text { A los } 1 \mathrm{~h} .17 \mathrm{~min} \text { se alconzó un valor medio } \\
\Delta T=140^{\circ}(*) \text { y la inestabilidad mecánica }\end{array}$ & RF 60 & 60 \\
\hline $\begin{array}{l}\text { FORJADO CADIZ } \\
\text { Nervios de } 0,12 \mathrm{~m} \text {. de oncho } \\
\text { coda } 0,60 \mathrm{~m} \text {. Copo de com- } \\
\text { presion de } 0,05 \mathrm{~m} \text {. Recubri - } \\
\text { miento de las ormaduras } \\
7,5 \mathrm{~cm} \text {. } \\
\text { Ploco de yeso- carton de } \\
10 \mathrm{~mm} \text {. }\end{array}$ & $\begin{array}{l}L=3,68 \mathrm{~m} \\
b=1,60 \mathrm{~m} \\
h=0,21 \mathrm{~m}\end{array}$ & $0,400 \mathrm{t} / \mathrm{m}^{2}$ & $\begin{array}{l}\text { A las } 1 \mathrm{~h} .36 \mathrm{~min} \text {. Se olconzó un } \\
\Delta T=180^{\circ}(*) \text { prolongándose el ensayo hasto } \\
\text { las } 2 \mathrm{~h} .15 \mathrm{~min} \text {. } \\
\text { Flecha final en centro vano }=2,5 \mathrm{~cm}\end{array}$ & RF 90 & 120 \\
\hline $\begin{array}{l}\text { FORJADO D.-P.I } \\
\text { Copo de } 1,5 \mathrm{~cm} \text { de yeso }\end{array}$ & $\begin{array}{l}L=3,68 \mathrm{~m} \\
b=1,60 \mathrm{~m} \\
h=0,145 \mathrm{~m}\end{array}$ & $0,150 t / \mathrm{m}^{2}$ & $\begin{array}{l}\text { A las } 3 \mathrm{~h} \text {. } 40 \mathrm{~min} \text {. se alcanzó } \\
\Delta \mathrm{T}=180^{\circ}(*) \text { y el ensayo se dió por } \\
\text { terminado. } \\
\text { Flecha tinal en centro vano }=11,5 \mathrm{~cm}\end{array}$ & RF 180 & 180 \\
\hline (*) VALORES MEDIC & IDOS EN TERM & PARES EN L & A CARA NO EXPUESTA AL FUEGO & & \\
\hline
\end{tabular}


racteristicas similares a los anteriores. En ambos casos las muestras se situaron en la boca de los hornos con una cara expuesta al fuego y se sellaron con lana mineral las uniones entre ellas y el horno.

Los ensayos comenzaron con la aplicación de cargas que producian en los elementos constructivos estados tensionales similares a los correspondientes a su situación de servicio en los edificios (tabla 1). Los muros fueron sometidos a cargas centradas de compresión aplicadas mediante dos cilindros hidráulicos a través de una viga metálica que repartia la carga uniformemente en todo su borde superior (fig. 1). En los forjados, que estaban apoyados en sus bordes más cortos sobre muros de ladrillos refractarios, se introdujeron cargas lineales a todo lo ancho del elemento situadas aproximadamente a tercios de la luz con un valor tal que producian el mismo momento flector máximo que las cargas equivalentes uniformemente repartidas (fig. 2).

Posteriormente, las muestras fueron sometidas al programa térmico normalizado (2) controlado automáticamente por ordenador, registrándose de forma continua la temperatura en el horno con seis termopares emplazados en diferentes puntos de su interior.

Durante el ensayo se median, de forma también continua, las temperaturas de la cara no expues-

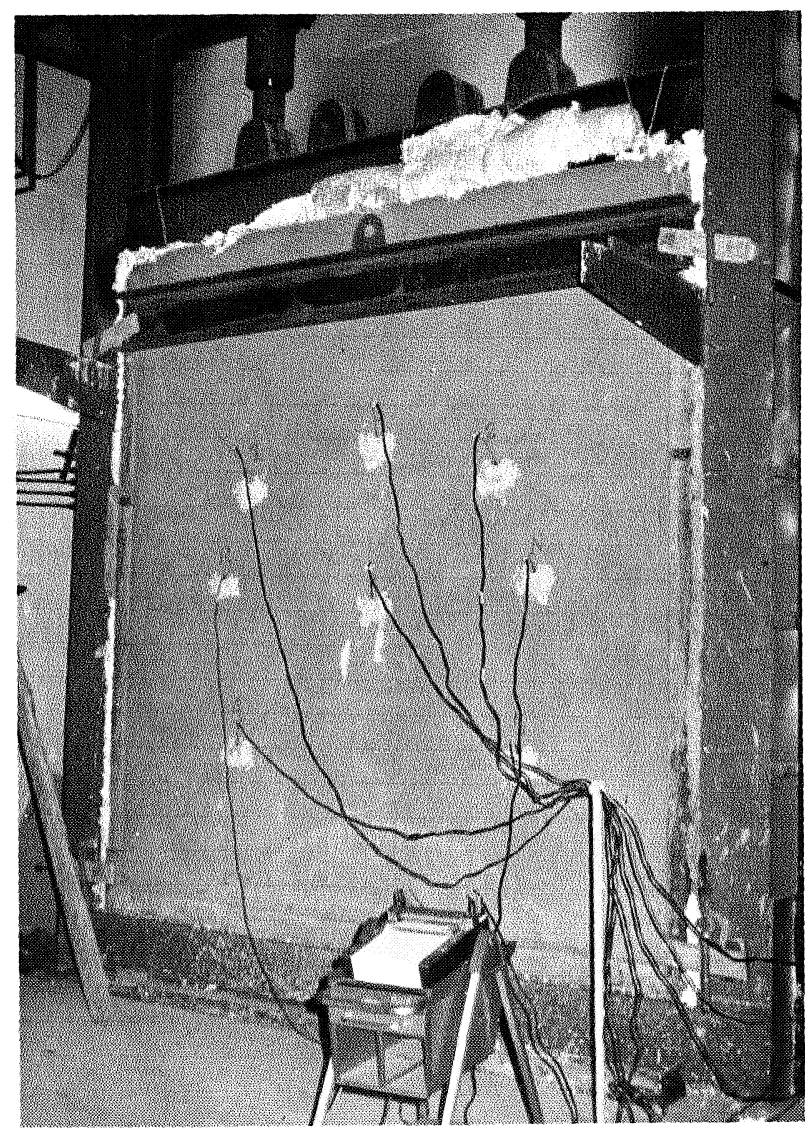

Fig. 1. - Ensayo de un muro D-P II bajo carga en el horno vertical.

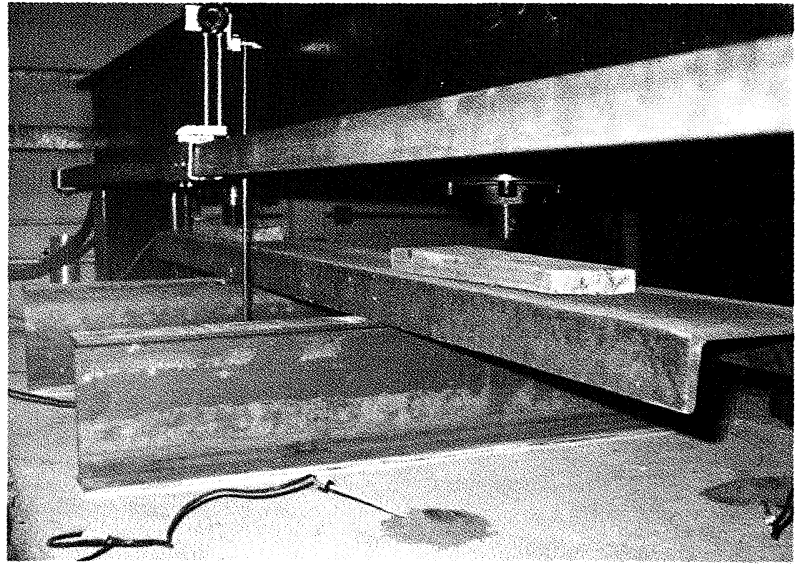

Fig. 2. - Ensayo de un forjado de hormigón bajo carga en el horno horizontal.

ta al fuego de los forjados y los muros mediante la colocación de 5 u 8 termopares respectivamente. La estanquidad a las llamas se controlaba visualmente y mediante la aproximación de un algodón a 20 ó $30 \mathrm{~mm}$ de cada fisura durante un tiempo de 10 a 30 segundos controlándose que dicho algodón no iniciaba la combustión.

En los forjados se llevó a cabo un seguimiento de la evolución de la flecha en centro del vano durante la acción del fuego lo que permitió conocer su velocidad de variación y el valor máximo alcanzado.

\section{RESULTADOS}

En todas las muestras ensayadas se perdió en primer lugar la condición de aislamiento térmico (incremento de temperatura en la cara no expuesta de $1800^{\circ}$ en un punto o de $1400^{\circ} \mathrm{C}$ como valor medio en toda ella). Sólo en el caso del muro D-P I sin revestimiento de yeso se alcanzó también de forma casi simultánea su inestabilidad mecánica. En los otros casos el ensayo se detuvo por mutuo acuerdo cuando todavia se mantenian las condiciones restantes.

En la parte central de la tabla 1 se resumen los resultados de los ensayos y en la misma tabla se indica también la clasificación de los elementos estructurales del sistema deducida de estos ensayos, señalando su «resistencia al fuego", tiempo en minutos del mantenimiento de todas las condiciones exigidas y el valor "parallamas", tiempo en minutos del mantenimiento de todas las condiciones excepto la del aislamiento térmico.

Durante la ejecución de estos ensayos se llevó a cabo una inspección visual de las muestras y las observaciones efectuadas asi como los resultados obtenidos se recogieron en los informes escritos de cada uno de ellos. 


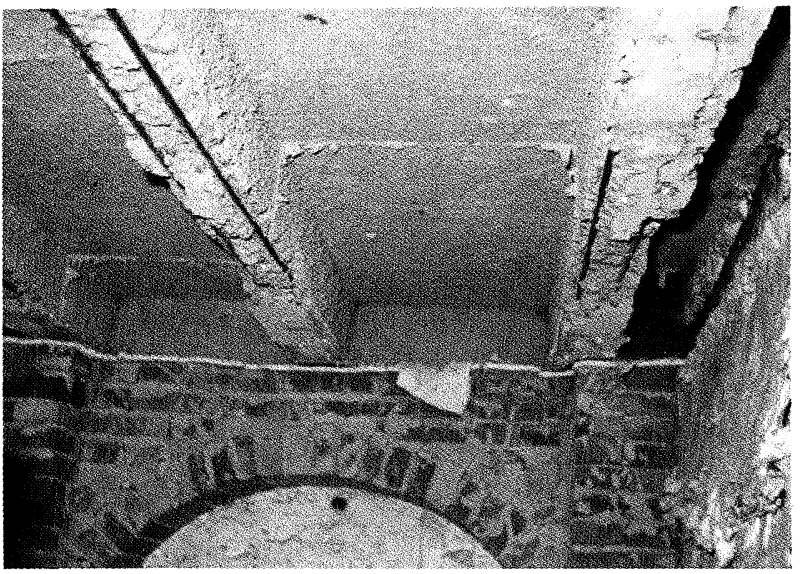

Fig. 3. - Aspecto de la cara inferior del forjado de hormigón después de la realización del ensayo.

Tanto los muros como los forjados fueron examinados con posterioridad a la realización de estos ensayos para conocer con más detalle el efecto sobre ellos de la acción del fuego. La fig. 3 muestra el aspecto del forjado Cádiz ensayado, por su cara inferior apreciándose la pérdida del yeso-cartón y de los aligeramientos de poliestireno, asi como la rotura del recubrimiento de hormigón de la cara inferior de los nervios del forjado, en la zona que no tenian armaduras transversales.

\section{CONCLUSIONES}

Estos ensayos han permitido clasificar los elementos de este sistema constructivo atendiendo a su comportamiento frente al fuego. De los valores obtenidos se deduce la viabilidad de su empleo como elementos estructurales y de cerramiento de edificios de dos y cinco plantas con muros D-P I y D-P II respectivamente, asi como de su empleo como elementos de cerramiento de edificios de mayor altura con estructuras convencionales.

En la tabla 2 se resumen los valores de resistencia al fuego de algunos elementos constructivos convencionales (1). Comparando las tablas 1 y 2 se observa que los elementos que forman el sistema Dragados-Plastbau tienen valores de resistencia al fuego de un orden de magnitud simi-
TABLA 2

Valores de la resistencia al fuego de algunos elementos constructivos (NBE-CPI-82)

\begin{tabular}{|c|c|c|c|c|c|}
\hline ELEMENTO CONSTRUCTIVO & & $\begin{array}{l}\text { CAPA } \\
1,5 \mathrm{~cm} .\end{array}$ & $\begin{array}{l}A \text { DE } \\
\text { YESO }\end{array}$ & $\begin{array}{l}\text { CAPA } \\
1,0 \mathrm{~cm}\end{array}$ & $\begin{array}{l}\text { DE } \\
\text { YESO }\end{array}$ \\
\hline $\begin{array}{l}\text { MURO DE LADRILLO } \\
\text { Macizo de } 11,5 \mathrm{~cm} \text {. de espesor } \\
\text { Hueco de } 11,5 \mathrm{~cm} \text {. de espesor }\end{array}$ & $\begin{array}{l}\text { RF. } 120 \\
\text { RF. } 90\end{array}$ & $\begin{array}{l}\text { RF. } \\
\text { RF. }\end{array}$ & $\begin{array}{l}180 \\
120\end{array}$ & & \\
\hline $\begin{array}{l}\text { MURO HORMIGON ARMADO } \\
\text { Canto } 16 \mathrm{~cm} \text {. y recubrimiento de } \\
\text { armaduras } 2,5 \mathrm{~cm} \text {. } \\
\text { Canto } 14 \mathrm{~cm} \text {. y recubrimiento de } \\
\text { armaduras } 7,5 \mathrm{~cm} \text {. }\end{array}$ & $\begin{array}{ll}\text { RF. } & 120 \\
\text { RF. } & 90\end{array}$ & $\begin{array}{l}\text { RF. } \\
\text { RF. }\end{array}$ & $\begin{array}{l}180 \\
120\end{array}$ & & \\
\hline $\begin{array}{l}\text { LOSA MACIZA HORMIGON ARMADO } \\
\text { Canto } 12 \mathrm{~cm} \text {. y recubrimiento de } \\
\text { ormaduros } 3,0 \mathrm{~cm} \text {. } \\
\text { Canto } 15 \mathrm{~cm} \text {. y recubrimiento de } \\
\text { armaduros } 5,5 \mathrm{~cm} \text {. }\end{array}$ & $\begin{array}{l}\text { RF. } 90 \\
\text { RF. } 180\end{array}$ & & & $\begin{array}{l}\text { RF. } \\
\text { RF. }\end{array}$ & $\begin{array}{l}120 \\
240\end{array}$ \\
\hline $\begin{array}{l}\text { FORJADO DE VIGUETAS Y BOVEDILLA } \\
\text { Canto } 17,5 \mathrm{~cm} \text {; ancho nervio } 10 \mathrm{~cm} \text {; } \\
\text { recubrimiento armaduro } 5,5 \mathrm{~cm} \text {. } \\
\text { Conto } 14 \mathrm{~cm} \text {; ancho nervio } 8 \mathrm{~cm} \text {; } \\
\text { recubrimiento ormadura } 3,0 \mathrm{~cm} \text {. }\end{array}$ & $\begin{array}{l}\text { RF. } 180 \\
\text { RF. } 90\end{array}$ & RF. 1 & 120 & & \\
\hline
\end{tabular}

lar al de elementos de hormigón o cerámica empleados en construcción.

\section{BIBLIOGRAFIA}

1. MOPU: "Condiciones de protección contra incendios en los edificios». Norma NBE-CPI-82, 1982.

2. IRANOR: «Ensayo de la resistencia al fuego de las estructuras y elementos de construcción». Norma UNE 23093-81, 1981.

3. ELVIRA, L. M. y JIMENEZ, F. J.: “Comportamiento al fuego de materiales y estructuras". Monografia n.o 37, Ministerio de Agricultura, Pesca y Alimentación, 1982.

4. JIMENEZ, F. J.: «Estructuras de madera laminada encolada. Su resistencia al fuego. Informatización de los ensayos". 1er Encuentro Internacional de Centros de Investigación del Fuego, ITSEMAP, Avila, 1986.

5. INTERNATIONAL STANDARD ORGANIZATION (ISO): «Fire-resistance tests-Elements of Building Construction», ISO 834 (1975). 